\title{
Covering the Cover
}

Critical flicker frequency test for diagnosing minimal hepatic encephalopathy in patients with cirrhosis

Minimal hepatic encephalopathy (MHE) is a well-described complication of cirrhosis affecting up to twothirds of patients. The initial symptoms of MHE may vary from reduced reaction times to coordination and functional problems, resulting in a reduced quality of life. Moreover, these patients are more likely to develop overt hepatic encephalopathy. Neuropsychometric tests can be helpful for diagnosing MHE; however, the definitive diagnosis is not straightforward. Recent research has suggested that the critical flicker frequency (CFF) may be a valuable tool to accurately identify MHE patients. In the article "Critical flicker frequency test for diagnosing minimal hepatic encephalopathy in patients with cirrhosis", Özel Coşkun et al. utilized the CFF test in 70 cirrhotic patients who were predominantly viral hepatitis-induced. Their findings showed that $41.4 \%$ and $35.7 \%$ of the patients had MHE by the CFF and psychometric tests, respectively. By combining both methods and setting the CFF cutoff at $39 \mathrm{~Hz}$, the researchers suggested that the diagnostic sensitivity would improve. See page 155.

\section{Pregnancy does not affect the fecal calprotectin con- centration in healthy pregnant women}

Fecal calprotectin has been increasingly used for diagnosing and monitoring inflammatory bowel disease. The paper by Bálint et al. in this issue of the Turkish Journal of Gastroenterology adds to the mounting evidence that fecal calprotectin and CRP may be utilized for diagnosing inflammatory bowel disease relapses in pregnancy. In this study, pregnancy did not alter fecal calprotectin concentrations unless it was associated with IBD. However, further prospective studies are needed to evaluate the use of calprotectin in pregnancy. See page 171.

Squamous cell papilloma of the esophagus: Analysis for the presence of human papilloma virus

Squamous cell papillomas are rare epithelial tumors that may progress to esophageal dysplasia and can- cer. In the present issue of the journal, Tiftikçi et al. published a human papilloma virus genotyping study on 38 patients with esophageal squamous cell papillomas. The papillomas were predominantly located at the middle and distal regions of the esophagus. The most striking data from this paper were the high number of patients (7 of 38) who tested positive for the human papilloma virus. Furthermore, their data confirmed the presence of highly oncogenic genotypes in four patients. Currently, the follow-up process for patients infected with oncogenic human papilloma virus is not clear. However, these findings may create further opportunities in this area and add more value to human papilloma virus genotyping in esophageal squamous cell papillomas. See page 176.

\section{Risk factors for bloating and its association with common gastrointestinal disorders in a sample of Iranian adults}

In the present issue of the journal, Keshteli et al. have addressed risk factors for bloating and associated gastrointestinal disorders in a large Iranian population. In this cross-sectional multicenter study, the Rome III questionnaire was administered to a remarkably large cohort of 4763 participants. The bloating severity and psychological assessment were investigated. Functional bloating was observed as a frequent condition and was defined as a recurrent feeling of bloating at least three days a month for the past three months in fewer than $20 \%$ of the healthy subjects. Functional bloating was associated with female gender and having a university degree, a high body mass index, and anxiety. Moreover, functional bloating indicated the presence of functional bowel disorders with a PPV and NPV of $93 \%$ and $80 \%$, respectively. For instance, $83 \%$ of the patients with IBS reportedly had bloating. Thus, this study showed a high prevalence of functional bloating in Iranian adults. This could be due to geographic factors and the ethnic background; however, this study warrants further investigations in diverse populations. See page 179. 
Evaluation of local tumor residue after percutaneous radiofrequency ablation therapy for hepatocellular carcinomas

The incidence of hepatocellular carcinomas (HCCs) has risen worldwide over the last decades, and major therapeutic advances have been achieved for early-stage HCCs. A local tumor residue after curative treatments may imply a poor prognosis. A retrospective study by Hamanaka et al. from Japan in this issue of the journal evaluated the efficacy of $\mathrm{CO}_{2}$ - and Sonazoid-enhanced ultrasonography (US) along with dynamic CT in detecting a local tumor residue after percutaneous radiofrequency (RF) ablation therapy. The study investigated 121 patients with HCCs; tumor residues were detected in 22 patients. The sensitivities of $\mathrm{CO}_{2}$-enhanced US, Sonazoid-enhanced US, and conventional US were $100 \%, 86 \%$, and $77 \%$, respectively. This study clearly favored the use of $\mathrm{CO}_{2}$-enhanced US in comparison to Sonazoid-enhanced US to detect tumor residues after percutaneous RF ablation therapy, particularly for deep-seated lesions. In summary, these outcomes are very appealing; follow-up with better imaging modalities may improve the standard care for HCCs, which can be of great value to hepatologists, oncologists, and surgeons. See page 197.

\section{Predictive factors for neoplastic gallbladder polyps: Out-} comes of $\mathbf{2 7 8}$ patients

Gallbladder polyps are commonly encountered in patients who have undergone an abdominal ultrasound examination. Due to the extremely malignant potential of gallbladder carcinomas, a timely discrimination of benign and neoplastic polyps is essential. This issue of the journal includes the study by
Terzioğlu et al. who examined the contribution of predictive factors in neoplastic gall bladder polyps. In this single-center retrospective study, the authors concluded that the size of the polyp and the sessile morphology are independent predictors of neoplastic gallbladder polyps. Therefore, large-sized polyps and the sessile morphology should be considered for timely surgery. See page 202.

Can C-reactive protein levels increase the accuracy of the Ranson score in predicting the severity and prognosis of acute pancreatitis? A prospective cohort study

The development of a prognostic scoring system in acute pancreatitis (AP) has received considerable attention. In this issue of the journal, a study has demonstrated the growing efforts for studying the assessment of severity in AP. A rigorous clinical study by Başak et al. assessed the efficacy of the Ranson criteria and CRP level for predicting the severity and mortality of AP in 638 patients. The combined use of a Ranson score of $\geq 3$ and CRP level of $\geq 150 \mathrm{mg} / \mathrm{L}$ predicted severe AP with a specificity of $97.1 \%$. On the other hand, when a Ranson score of $\geq 3$ or CRP level of $\geq 150 \mathrm{mg} / \mathrm{L}$ was separately used to assess severe $A P$, the sensitivity was $93.5 \%$. In this study, the sensitivity and specificity of the Ranson criteria to detect severe AP were $78.2 \%$ and $90.9 \%$, respectively; therefore, the addition of CRP elevation clearly improves the sensitivity of the method. See page 207.

\section{Müjdat Zeybel \\ Covering the Cover Editor}

